\title{
STRONG RATIO LIMIT PROPERTY FOR $R$-RECURRENT MARKOV CHAINS
}

\author{
WILLIAM E. PRUITT ${ }^{1}$
}

1. Introduction. For every non-negative integer $n$ let $p_{i j}^{(n)}$ be the $n$-step transition probabilities of an irreducible aperiodic Markov chain, $i, j=0,1,2, \cdots$. The chain has the strong ratio limit property (SRLP) if there are positive constants $\gamma, \pi_{i}, \tau_{i}, i=0,1,2, \cdots$, such that

$$
\lim _{n \rightarrow \infty} \frac{p_{i j}^{(m+n)}}{p_{k h}^{(n)}}=\gamma^{m} \frac{\tau_{i} \pi_{j}}{\tau_{k} \pi_{h}}, \quad m=0, \pm 1, \pm 2, \cdots .
$$

Let $P_{i j}(z)=\sum_{n} p_{i j}^{(n)} z^{n}$, and let $R_{i j}$ denote the radius of convergence of this power series. Vere-Jones $\left[8\right.$, p. 14] proved that $R_{i j}$ is independent of $i$ and $j$ and that the series $P_{i j}(R)$ diverge or converge together. In the first case the chain is called $R$-recurrent and in the other $R$-transient.

The principal result of this paper is that, for an $R$-recurrent chain, if

$$
\limsup _{n \rightarrow \infty} \frac{p_{00}^{(n d+d)}}{p_{00}^{(n d)}} \leqq R^{-d}
$$

for some positive integer $d$, then SRLP holds. It is also shown that the inequality (1.2) is always satisfied in a reversible chain. When $R=1$, the results given here reduce to those of Orey [7] for recurrent chains, while for $R>1$ they imply SRLP for a class of transient chains. (The definition of SRLP given by Orey required that $\gamma=1$ and that $\tau_{i}$ be independent of $i$ since he was dealing with recurrent chains. The definition given here is the more natural one for transient chains.)

The proofs given here were suggested by those used by Orey in the recurrent case. Although the proof given for the lemma is shorter than Orey's, the fundamental difference here is an analytic proof of Theorem 1 to replace the previous probabilistic one. ${ }^{2}$

Received by the editors October 3, 1963.

1 This work was partially supported by the Air Force Office of Scientific Research.

2 Note added in proof. J. F. C. Kingman has observed that Theorem 1 may also be obtained as a consequence of Orey's theorem for recurrent chains by making an appropriate transformation on the transition probability matrix. This transformation is described in $[8, p .20]$. Since the primary point of interest here is the analytic proof which was suggested by the situation when $R>1$, the proof has been left in this setting even though it could have been reduced to the case of $R=1$ by the transformation technique. 
2. The ratio limit theorem. Let ${ }_{k} p_{i j}^{(n)}$ be the probability, starting from $i$, of being at $j$ at step $n$ without visiting $k$ at steps $1,2, \cdots$, $n-1$. Denote by ${ }_{k} P_{i j}(z)$ the generating function of this sequence.

LEMмA. If the chain is $R$-recurrent and

$$
\limsup _{n \rightarrow \infty} \frac{p_{00}^{(n+1)}}{p_{00}^{(n)}} \leqq R^{-1}
$$

then SRLP holds.

Proof. For $n \geqq-m+2$, consider the decomposition [1],

$$
p_{i j}^{(m+n)}=0 p_{i j}^{(m+n)}+\sum_{\nu=1}^{m+n-1} \sum_{\mu=1}^{m+n-\nu} 0 p_{i 0}^{(\nu)} 0 p_{0 j}^{(\mu)} p_{00}^{(m+n-\nu-\mu)} .
$$

Assume that $m \leqq 0$, divide by $p_{00}^{(n)}$ and let $n \rightarrow \infty$. (If $m>0, p_{00}^{(m+n)}$ should be used instead of $p_{00}^{(n)}$.) The result is

$$
\begin{aligned}
\lim _{n \rightarrow \infty} \inf _{n \rightarrow \infty} \frac{p_{i j}^{(m+n)}}{p_{00}^{(n)}} & \geqq \sum_{n=1}^{\infty} \sum_{\mu=1}^{\infty} 0 p_{i 0}^{(n)} 0 p_{0 j}^{(\mu)} R^{p+\mu-m} \\
& =R^{-m}{ }_{0} P_{i 0}(R)_{0} P_{0 j}(R) .
\end{aligned}
$$

Fix $i$ and $j$ and choose $\alpha$ and $\beta$ so that $p_{0 i}^{(\alpha)}$ and $p_{j 0}^{(\beta)}$ are positive and $\alpha+\beta \geqq-m$. For $n \geqq-m$,

$$
p_{00}^{(n+m+\alpha+\beta)}=\sum_{k, h} p_{0 k}^{(\alpha)} p_{k h}^{(m+n)} p_{h 0}^{(\beta)} .
$$

Divide by $p_{00}^{(n)}$ and let $n$ go to infinity along a subsequence chosen so that $p_{i j}^{(m+n)} / p_{00}^{(n)}$ approaches its lim sup. The lim inf of the left-hand side is no larger than the corresponding lim sup when not restricted to the subsequence. By (2.1) this is bounded above by $R^{-m-\alpha-\beta}$. The lim inf's of the individual terms on the right will be bounded below by the lower bounds obtained in the first part of the proof. Therefore

$$
\begin{aligned}
R^{-m-\alpha-\beta} \geqq & \sum_{k, h} p_{0 k}^{(\alpha)} R^{-m}{ }_{0} P_{k 0}(R){ }_{0} P_{0 h}(R) p_{h 0}^{(\beta)} \\
& +p_{0 i}^{(\alpha)}\left(\limsup _{n \rightarrow \infty} \frac{p_{i j}^{(m+n)}}{p_{00}^{(n)}}-\liminf _{n \rightarrow \infty} \frac{p_{i j}^{(m+n)}}{p_{00}^{(n)}}\right) p_{j 0}^{(\beta)} .
\end{aligned}
$$

To complete the proof it suffices to observe that

$$
\sum_{k} p_{0 k}^{(\alpha)}{ }_{0} P_{k 0}(R)=R^{-\alpha} \text { and } \sum_{h 0} P_{0 h}(R) p_{h 0}^{(\beta)}=R^{-\beta} \text {. }
$$


For $R=1$, these are simply a row sum of $P^{\alpha}$ being one, and one component of Derman's [2] equation for the stationary measure. The generalizations for $R>1$ are due to Vere-Jones [8, p. 19].

Let $f_{0}=0, u_{0}=1 ; f_{n}={ }_{0} p_{00}^{(n)}, u_{n}=p_{00}^{(n)}, n \geqq 1$. These sequences are connected by the familiar renewal relation

$$
u_{n}=\sum_{\nu=1}^{n} f_{\nu} u_{n-\nu}, \quad n \geqq 1 \text {. }
$$

Their generating functions are then related by $F(z)=1-1 / U(z)$ for $|z|<R$. It follows that, in an $R$-recurrent chain, $F(R)=1$.

THEOREM 1. If the chain is R-recurrent and, for some positive integer $d$,

$$
\limsup _{n \rightarrow \infty} \frac{u_{(n+1) d}}{u_{n d}} \leqq R^{-d}
$$

then SRLP holds.

Proof. For $j \geqq 0$, define

$$
x_{j}=R^{-j} \liminf _{n \rightarrow \infty} \frac{u_{n d-j}}{u_{n d}}, \quad y_{j}=R^{-j} \limsup _{n \rightarrow \infty} \frac{u_{n d-j}}{u_{n d}}
$$

and notice that

$$
x_{j+d} \geqq R^{-j} \liminf _{n \rightarrow \infty} \frac{u_{n d-d-j}}{u_{n d-d}} R^{-d} \liminf _{n \rightarrow \infty} \frac{u_{n d-d}}{u_{n d}} \geqq x_{j} .
$$

By (2.2), $u_{n d-j}=\sum_{\nu=1}^{n d-j} f_{\nu} u_{n d-j-\nu}$. Dividing by $R^{i} u_{n d}$ and letting $n \rightarrow \infty$ yields

$$
x_{j} \geqq \sum_{\nu=1}^{\infty} f_{\nu} R^{\nu} x_{j+\nu}
$$

The inequality (2.3) implies that the sequence $\left\{x_{j}\right\}$ attains its minimum value $c$ for some index $k$ between 0 and $d$. Since $F(R)=1,(2.4)$ then shows that $x_{k+\nu}=c$ for all $\nu$ such that $f_{\nu}>0$. In fact, by iterating the inequality (2.4) and using the aperiodicity assumption we see that $x_{j}=c$ for all sufficiently large $j$. It now follows from (2.3) that the minimum is attained for all $j$. Since $x_{0}=1, x_{j}=1$ for all $j$.

Fix an index $i$ and choose a subsequence of $n$ so that $u_{n d-i} / u_{n d}$ approaches its lim sup along this subsequence. In place of (2.4), we have, for $j<i$,

$$
y_{j} \geqq \sum_{\nu} f_{\nu} R^{\nu} x_{j+\nu}+f_{i-j} R^{i-j}\left(y_{i}-x_{i}\right)
$$


or $y_{j}-1 \geqq f_{i-j} R^{i-j}\left(y_{i}-1\right)$. Since $y_{0}=1, y_{i}=1$ for all $i$ such that $f_{i}>0$ or, again by iteration, $y_{i}=1$ for all sufficiently large $i$. Thus we certainly have $d+1$ consecutive values of $j$ such that $\lim \left(u_{n d-j} / u_{n d}\right)=R^{j}$ as $n \rightarrow \infty$. This implies $\lim \left(u_{n+1} / u_{n}\right)=R^{-1}$ and the lemma completes the proof.

3. Reversible processes. The transition matrix is said to be reversible if and only if there is a sequence $\left\{\rho_{i}\right\}$ of positive constants such that $\rho_{i} p_{i j}=\rho_{j} p_{j i}$ for every $i$ and $j$.

THEOREM 2. Reversibility implies (1.2) (and therefore SRLP if the chain is also R-recurrent).

Proof. Kendall [6] has shown that for a reversible chain

$$
u_{n}=\int_{-1}^{1} x^{n} d \psi(x), \quad n=0,1,2, \cdots
$$

where $\psi$ is a nondecreasing bounded function. Therefore

$$
2\left(u_{2 n+2} u_{2 n-2}-u_{2 n} u_{2 n}\right)=\iint x^{2 n-2} y^{2 n-2}\left(x^{2}-y^{2}\right)^{2} d \psi(x) d \psi(y) \geqq 0,
$$

so that $u_{2 n+2} / u_{2 n}$ is a nondecreasing sequence and must converge. The limit will be the reciprocal of the radius of convergence of the power series $\sum_{n} u_{2 n} z^{n}$. But this series will certainly converge for $|z|<R^{2}$ so that $\lim \left(u_{2 n+2} / u_{2 n}\right) \leqq R^{-2}$.

EXAmple 1 (simple random walk with a reflecting barrier). The state space is the non-negative integers and $p_{00}=q, p_{01}=p ; p_{i, i-1}=q$, $p_{i, i+1}=p, i \geqq 1$. If $p>1 / 2$, Karlin and McGregor [5] have observed that SRLP does not hold. The chain is necessarily $R$-transient and this is a case where $u_{2 n+1} / u_{2 n}$ and $u_{2 n+2} / u_{2 n+1}$ converge to distinct positive limits.

EXAMPLE 2 (unrestricted simple random walk). The state space is all the integers and $p_{i, i-1}=q, p_{i, i+1}=p$ for all $i$. This chain is $R$-recurrent but periodic so the SRLP cannot hold.

EXAMPLE 3. The state space is again all the integers and $p_{i, i-1}=q, p_{i i}=r, p_{i, i+1}=p$ for all $i, p+q+r=1$, with $p, q, r>0$. This chain is aperiodic and $R$-recurrent so it has the SRLP.

\section{BIBLIOGRAPHY}

1. K. L. Chung, Markov chains with stationary transition probabilities, Springer, Berlin, 1960.

2. C. Derman, $A$ solution to a set of fundamental equations in Markov chains, Proc. Amer. Math. Soc. 5 (1954), 332-334.

3. W. Feller, An introduction to probability theory and its applications, Wiley, New York, 1950. 
4. A. Garsia, S. Orey and E. Rodemich, Asymptotic behavior of successive coeffcients of some power series, Illinois J. Math. 6 (1962), 620-629.

5. S. Karlin and J. McGregor, Random walks, Illinois J. Math. 3 (1959), 66-81.

6. D. G. Kendall, Unitary dilations of Markov transition operators, and the corresponding integral representation for transition-probability matrices, Harold Cramer Volume (U. Grenander, Ed.), Almquist and Wiksell, Stockholm, 1960; pp. 139-161.

7. S. Orey, Strong ratio limit property, Bull. Amer. Math. Soc. 67 (1961), 571-574.

8. D. Vere-Jones, Geometric ergodicity in denumerable Markov chains, Quart. J. Math. Oxford Ser. (2) 13 (1962), 7-28.

UnIVERSITY OF MinNESOTA

\title{
A REPRESENTATION THEOREM FOR CONTINUOUS FUNCTIONS OF SEVERAL VARIABLES
}

\author{
DAVID A. SPRECHER ${ }^{1}$
}

Let $E^{n}$ denote the $n$-dimensional unit cube in Euclidean space; designate the closed unit interval, $[0,1]$, by $E$. We prove in this note the following

THEOREM. For any natural number $n, n \geqq 2$, there exist real, monotonic increasing functions, $h^{p}(x), 1 \leqq p \leqq n$, dependent on $n$, and having the following properties:

(i) The function

$$
\sum_{1 \leq p \leq n} h^{p}\left(x_{p}\right)
$$

separates all points of $E^{n}$ :

$$
\sum_{1 \leq p \leq n} h^{p}\left(x_{p}\right) \neq \sum_{1 \leq p \leq n} h^{p}\left(y_{p}\right)
$$

unless $x_{p}=y_{p}$ for all admitted values of $p$.

(ii) Every continuous function of $n$ variables, $f\left(x_{1}, \cdots, x_{n}\right)$, with domain $E^{n}$, can be represented in the form

$$
f\left(x_{1}, \cdots, x_{n}\right)=g\left[\sum_{1 \leq p \leq n} h^{p}\left(x_{p}\right)\right] .
$$

Clearly, the function $g$ will, in general, be discontinuous.

Received by the editors September 16, 1963.

1 This note is part of the author's Ph.D. thesis (University of Maryland, 1963, directed by A. Douglis); its research was supported by the United States Air Force through the AFOSR under Contract No. AF 49(638)-590. 